\title{
Screening of the College Students at Clinical High Risk for Psychosis in China: A Multicenter Epidemiological Study
}

JiaXin Wu

Tongji Hospital of Tongji University, Tongji University School of Medicine

XiangYun Long

Tongji Hospital of Tongji University, Tongji University School of Medicine

Fei Liu

Tongji Hospital of Tongji University

AnSi Qi

Tongji Hospital of Tongji University

Qi Chen

Tongji Hospital of Tongji University

\section{XiaoFeng Guan}

Tongji Hospital of Tongji University

Qiong Zhang

Tongji Hospital of Tongji University

\section{YuHong Yao}

Tongji University School of Medicine

JingYu Shi

Tongji University School of Medicine

\section{ShiPing Xie}

Nanjing Brain Hospital

\section{Wei Yan}

Nanjing Brain Hospital

\section{MaoRong Hu}

First Affiliated Hospital of Nanchang University

\section{Xin Yuan}

First Affiliated Hospital of Nanchang University

Jun Tang

First Affiliated Hospital of Nanchang University

\section{SiLiang Wu}

First Affiliated Hospital of Nanchang University

TianHong Zhang 
Shanghai Mental Health Center

\section{JiJun Wang}

Shanghai Mental Health Center

Zheng Lu ( $\nabla$ luzheng@tongji.edu.cn )

Tongji University School of Medicine

\section{Research article}

Keywords: Clinical high risk, Ultra-high risk, Early detection, College students, Epidemiology

Posted Date: August 28th, 2020

DOI: https://doi.org/10.21203/rs.3.rs-62868/v1

License: (c) (i) This work is licensed under a Creative Commons Attribution 4.0 International License. Read Full License

Version of Record: A version of this preprint was published at BMC Psychiatry on May 17th, 2021. See the published version at https://doi.org/10.1186/s12888-021-03229-8. 


\section{Abstract \\ Background}

To investigate a 3-stage screening procedure and explore the clinical features of subjects at Clinical High Risk (CHR) for psychosis in a representative sample of Chinese college students.

\section{Methods}

An epidemiological survey of the prevalence of CHR syndrome in Chinese college students that was selected by stratified random sampling from Shanghai, Nanjing and Nanchang cities was done followed a 3-stage procedure. Participants was initially screened with the Prodromal Questionnaire-brief version (PQ-B), and whose distress score of PQ-B exceeded 24 would be invited to a telephone assessment using the subscale for positive symptoms of the Scale of Prodromal Symptoms (SOPS)/Structured Interview for Prodromal Syndromes (SIPS). Lastly, participants who scored 3 or higher in any item of the subscale would be administered with the SIPS interview conducted by trained researchers to confirm the diagnosis of CHR syndrome.

\section{Results}

23063 college students completed the survey during September 2017 to October 2018.72 students were diagnosed as $\mathrm{CHR}$ subjects, and the detection rate in the total sample was $0.3 \%$. The peak age range for the first diagnosis of CHR was 17 to 20 years. 13 and 46 were set as the cut-off points of PQ-B total score and distress score to balance the greatest sensitivity and specificity. Binary logistic regression revealed that 5 items in PQ-B showed significant distinction for detecting CHR subjects.

\section{Conclusions}

The 3-stage screening method can be utilized in the detection of CHR subjects for psychosis in general population, during which delusional ideas, perceptual abnormalities and suspiciousness deserve great attention.

\section{Background}

Since being proposed by Sullivan in 1927, the notion of early detection and preventive intervention of psychosis syndromes has received increased attention in the recent two decades [1-4]. As a series of studies of first-episode psychosis suggests that longer duration of untreated psychosis is significantly correlated with worse treatment response and prognosis[5], global researchers and clinicians have reached a consensus that early detection and prevention is central to contain the development and burden of psychosis. They started to investigate whether there is an earlier period in the development of 
psychosis that can be detected before the break, and then gave birth to the concept of ultra-high risk (UHR) or clinical high risk (CHR) for psychosis[6, 7]. CHR subjects refer to individuals who are identified as having nonspecific symptoms (e.g. cognitive symptoms) and attenuated or intermittent psychotic symptoms, or having asymptomatic genetic risk, but do not meet criteria for a syndromal psychosis, and thereby they are considered to be at increased risk for developing psychotic disorders $[4,8]$. Fusar-Poli et al.[9] reported that $20-35 \%$ CHR subjects developed syndromal psychotic disorder in 2 years, and Zhang et al.[10] discovered that the transition rate at the first 2 years was $26.4 \%$ in a Chinese help-seeking sample, both of which indicated that the risk of developing psychosis in CHR subjects deserved serious attention.

To diagnose and assess symptoms of CHR, two categories of instruments based on two complementary criteria have been globally recognized: the UHR criteria that focus on attenuated or brief limited intermittent psychotic symptoms and genetic risk, and the basic symptoms criteria that focus on cognitive and perceptive symptoms. The UHR criteria were designed to detect the risk for developing a first-episode psychosis in 12 months[11]. The most recognized instruments developed from the UHR criteria include the Structured Interview for Psychosis-risk Syndromes (SIPS)[12] and the Comprehensive Assessment of At-Risk Mental States (CAARMS)[13]. While the basic symptoms criteria aimed at detect the risk for psychosis ideally before the appearance of functional impairments[11]. The most representative instruments are the Schizophrenia Proneness Instrument, Adult (SPI-A)[14] and Child \& Youth version (SPI-CY)[15]. Since the instruments listed above are based on the clinician's assessments through clinical interviews, several self-report scales have been established to simplify the screening of CHR individuals, such as Prodromal Questionnaire-Brief version (PQ-B)[16], Prodromal Questionnaire-16 items (PQ-16)[17], Prime Screen[18] and Early Psychosis Screener (EPS)[19, 20].

Among the instruments listed above, SIPS[21], PQ-B[22], and PQ-16[23] have been translated into Chinese version, which showed good reliability and validity in screen CHR individuals at clinical settings.

Notwithstanding, detection of CHR in China is still limited by its passivity that the clinical interviews and rating scales can almost only be approached in psychiatric clinics or wards, which indicates that only individuals with help-seeking behaviors can be tested, and doctors should always wait passively for the suspected cases. While some of them won't see a doctor (e.g. because of social discrimination, stigma or poor knowledge of psychosis) before the onset of psychosis or significant functional impairments[24]. In fact, most researches concerning CHR detection in China were conducted in help-seeking individuals[10, $25,26]$. Besides, as the risk age range of $\mathrm{CHR}$ is 15 to 22 years[27], studies carried out in non-clinical settings should pay more attention to students in university and high schools. Chen et al. established a two-stage screening method (PQ-16 followed by SIPS) in 579 Chinese university students who were enrolled in a mental health education course[23]. There were also two researches using self-report scales to explore the psychotic-like experiences (PLEs) in high-school students and university students in China[28, 29]. However, an epidemiological study of CHR detection conducted in a larger sample of college students with established psychosis-risk instruments still lacks in China. Therefore, we generalized a 3-stage method (consists of PQ-B, telephone interview and SIPS) for the detection of CHR individuals in college students from Shanghai, Nanjing and Nanchang in China. The present study aimed 
to investigate the screening procedure, and explore the characteristics of prodromal symptoms for psychosis in Chinese college students.

\section{Method}

\subsection{Participants}

The Research Ethics Committee at the Shanghai Mental Health Centre (SMHC), Shanghai, China approved this study in 2016. The participants were college students recruited by three research teams at three different cities: Tongji Hospital of Tongji University (TJH) at Shanghai, Nanjing Brain Hospital (NBH) at Nanjing, and The First Affiliated Hospital of Nanchang University (FAHNU) at Nanchang, from September 2017 to October 2018. Stratified random sampling was conducted at the level of school's level, including the first-tier universities, second-tier universities, third-tier universities and the higher vocational and technical colleges. Print version and online version of questionnaires were distributed to the selected sample of 27145 students. Since the written informed consents were not applicable in the online survey and telephone assessment, they were only obtained from the participants who received a face-to-face interview. Nevertheless, a detailed explanation of the study and informed consent were written in their native language in the beginning of the questionnaire, and participants were asked to complete the questionnaire only if they were voluntary. Similarly, the informed consents were orally made in the beginning of the telephone assessment to protect the basic rights of the participants. Eventually, 26 005 questionnaires were received, and 2942 questionnaires were removed because of data missing including demographic information and blank in items of the PQ-B. Thus, a sample of 23063 college students were investigated in this study. The age range of the investigated sample was 14 to 31 (mean = 18.4 years, S.D. $=1.2$ years), and $11028(47.8 \%)$ subjects were female.

\subsection{Instruments}

\subsubsection{The Prodromal Questionnaire-Brief version (PQ-B)}

The PQ-B[16] is a 21-item self-report scale designed to detect individuals at risk for psychosis. It was modified from the 92-item Prodromal Questionnaire[30] and focus on positive symptoms. Each item was marked with "Yes/No" by the participants according to the presence or absence of the corresponding experience in the past month. If an item was marked as "No", the item was rated as 0 ; if an item was marked as "Yes", a 5-point Likert scale (strongly disagree, disagree, neutral, agree, strongly agree) was used to rate the distress level of the symptom. The total score was defined as the number of the items endorsed, and the distress score was the sum of the distress level of the items endorsed.

Zhang et al[10] and Xu et al.[22] translated the original version of PQ-B into Simplified Mandarin Chinese, and reported good reliability and validity in a sample of help-seeking outpatients in China (Cronbach's alpha $=0.897$ ). They set the cutoff point for the total score and distress score of the Chinese-version PQ-B as 7 and 24 respectively. The sensitivity and specificity were reported to be $82.0 \%$ and $46.8 \%$ respectively 
when using the cutoff point for the distress score to screen CHR subjects. In the present study, the internal consistency of the Chinese-version PQ-B was excellent (Cronbach's alpha =0.928).

\subsubsection{The Structured Interview for Psychosis-risk Syndromes/Scale of Prodromal Syndromes (SIPS/SOPS)}

The SIPS/SOPS[12] were intended to define whether an individual met the CHR criteria through a clinician-administered, semi-structured clinical interview. The criteria outlined three types of PsychosisRisk Syndromes: Attenuated Positive Symptom Syndrome (APSS), Brief Intermittent Psychotic Syndrome (BIPS), and Genetic Risk and Deterioration Syndrome (GRDS). There are 19 items assessing 4 major symptom domains on the SIPS/SOPS, including Positive symptoms, Negative symptoms, Disorganized symptoms, and General Symptoms. Each item was rated from 1 to 6, according to the severity of the symptom. Rating of 3 to 5 indicates a prodromal range symptom, and 6 indicates a "severe and psychotic" symptom. Li et al. established the Chinese version of SIPS/SOPS, and it was demonstrated with good reliability and validity in assessing subjects with Psychosis-Risk Syndromes[21]. The internal consistency reliability of SIPS/SOPS in this study was good (Cronbach's alpha $=0.848$ ).

\subsubsection{Demographic characteristics}

Demographic characteristics that include name, sex, age, discipline, family history and contact information were measured with a questionnaire designed by the authors.

\subsection{Procedure}

A 3-stage screening procedure (see Fig. 1) was applied in this study. The screening results of the Chineseversion PQ-B were obtained from 23063 participants. According to Xu et al.[22], the cutoff point of the distress score of PQ-B was set as 24 in this study. 10330 (44.8\%) participants reached this cutoff point, and were invited to a telephone assessment. Since 666 participants were failed to be contacted (e.g. dead number, suspended service or didn't answer for three times) or declined to receive the telephone assessment, 9664 (41.9\%) participants were assessed by trained researchers, with the items listed in the subscale for positive symptoms of the SIPS/SOPS (P1-P5, Unusual Thought Content, Suspiciousness/Persecutory Ideas, Grandiosity, Perceptual Abnormalities/Hallucinations, and Disorganized Communication) to identify the possible presence of the prodromal or psychotic symptoms. If any item was rated as 3 or higher, the participant would be considered as positive in the telephone assessment. Thus, 1176 participants were invited to the face-to-face interviews, and 991 (4.3\%) participants eventually consented to receive the SIPS interviews administered by trained researchers. The participants that met any criterion of the Psychosis-Risk Syndromes (APSS, BIPS, GRDS) defined by SIPS were diagnosed as $\mathrm{CHR}$ individuals. All interviewers had received a professional training for SIPS prior to the study. The inter-rater reliability was good among the interviewers $(n=20, I C C=0.75)$.

\subsection{Statistical analyses}


The sample size of this study was estimated by the prevalence (p) of CHR syndrome, which was reported to be $0.3 \%$ in a young adults community[31]. Using the formula[32]:

$$
\mathrm{n}=\mathrm{Z}_{1-\alpha}^{2}(1-\mathrm{p}) / \mathrm{p} \epsilon
$$

with the relative error $(\varepsilon)$ set as $25 \%$, the sample size was calculated to be roughly 20500 . Considering a response rate of $80 \%$, we were required to survey 25625 individuals.

Using SPSS software (IBM Corp. Released 2017. IBM SPSS Statistics for Macintosh, Version 25.0. Armonk, NY: IBM Corp.), the group differences in age, the total score and distress score as well as each item score of the PQ-B were compared using Independent-samples t-tests, and the significance level was set at 0.05 (two-tailed). Likewise, Chi-square tests were used to compare sex between the two groups. Mann-Whitney $\mathrm{U}$ test was used to test whether there was a difference in disciplines between the two groups. Since $60.8 \%$ of the data of the family history were missing, family history was not described in the demographic characteristics. The internal consistency reliability of PQ-B and SOPS were described using the Cronbach's alpha. The receiver operating characteristic (ROC) analyses were performed to reset the cutoff points and to examine the sensitivity and specificity of PQ-B in detecting CHR subjects. The accuracy of the tests was described through the areas under the ROC curve (AUCs) with the $95 \%$ confidential interval $(\mathrm{Cl})$. A Spearman correlation analysis was used to investigate the association between each item score of PQ-B and the dichotomized SIPS diagnosis. A logistic regression analysis was conducted to explore the significant PQ-B items in indicating the CHR states based on the SIPS. For graphical presentation, SPSS software was utilized to draw the bar diagrams exhibiting the number of the CHR subjects by the age, as well as its distribution in female and male groups, and GraphPad Prism version 8.1.1 for Macintosh (GraphPad Software, San Diego, California USA, www.graphpad.com) was utilized to draw the bar-chart (mean and standard error of the mean) showing the differences in the PQ-B item scores between CHR and non-CHR subjects.

\section{Results}

\subsection{Demographic characteristics}

72 college students met the criteria for CHR states according to SIPS. The detection rate in the total sample was $0.3 \%$ (72/23063). The demographic characteristics of CHR individuals and non-CHR individuals were presented in Table 1. No significant difference in the mean age, sex, and disciplines was found between the two groups. 
Table 1

Demographic characteristics of the CHR and non-CHR individuals

\begin{tabular}{|lllll|}
\hline Variables & $\begin{array}{l}\text { CHR } \\
(\mathbf{n}=72)\end{array}$ & $\begin{array}{l}\text { Non-CHR } \\
(\mathbf{n}=22991)\end{array}$ & $\mathbf{t} / \mathbf{\chi}^{2} / \mathbf{U}$ & $\mathbf{p}$ \\
\hline Age mean (S.D.) & $18.3(1.5)$ & $18.4(1.2)$ & 0.38 & 0.707 \\
\hline Female (\%) & $38(52.8)$ & $10990(47.8)$ & 0.71 & 0.399 \\
\hline Disciplines & & & & \\
\hline Arts (\%) & $19(26.4)$ & $2502(10.9)$ & 751769.50 & 0.243 \\
\hline Humanities (\%) & $4(5.6)$ & $1100(4.8)$ & 751769.50 & 0.243 \\
\hline Social sciences (\%) & $3(4.2)$ & $2482(10.8)$ & 751769.50 & 0.243 \\
\hline Natural sciences (\%) & $3(4.2)$ & $3355(14.6)$ & 751769.50 & 0.243 \\
\hline Applied sciences (\%) & $40(55.6)$ & $12769(55.5)$ & 751769.50 & 0.243 \\
\hline Unclassified (\%) & $2(2.8)$ & $623(2.7)$ & 751769.50 & 0.243 \\
\hline Null (\%) & $1(1.4)$ & $160(0.7)$ & 751769.50 & 0.243 \\
\hline PQ-B total score mean (S.D.) & $18.0(4.4)$ & $8.5(7.1)$ & 18.22 & $<0.001$ \\
\hline PQ-B distress score mean (S.D.) & $66.0(22.1)$ & $24.6(21.1)$ & 16.64 & $<0.001$ \\
\hline
\end{tabular}

\subsection{Age of CHR subjects at identification}

The age distribution of CHR subjects was shown in Fig. 2. The frequency of CHR identified with SIPS in this sample peaked at young adulthood (17 to 20 years) and decreased with subsequent age. The peak age that met the CHR criteria in both males and females were 18 years old. While the age range of female subjects ( 15 to 26 years old) was broader than that of male subjects ( 15 to 21 years old).

\subsection{Evaluative value of $P Q-B$ in assessing $C H R$ individuals 3.3.1. The total score and distress score of PQ-B in detecting CHR}

Compared to non-CHR individuals, both the total score and distress score of PQ-B were significantly higher in CHR individuals (see Table 1). To examine the accuracy and to reset the cutoff points for Chinese college students, the diagnosis based on SIPS was set as the state variable, and the ROC analyses were performed for the PQ-B total score and distress score, whose AUCs were also calculated (see Fig. 2). Both the total score and distress score yielded significant value in assessing CHR individuals. The total score of PQ-B yielded an AUC of $0.842(95 \% \mathrm{Cl}: 0.806,0.877)$ for screening CHR, and the AUC of the distress score was 0.900 ( $95 \% \mathrm{Cl}: 0.863,0.937)$. At different cutoff points for the total score and 
distress score of $\mathrm{PQ}-\mathrm{B}$, the sensitivity and specificity values were calculated and presented in Table 2. The cutoff point of 13 for the total score, and 46 for the distress score balanced the greatest sensitivity and greatest specificity. For the total score, a cutoff point of 13 or higher possessed a sensitivity of $91.7 \%$, and a specificity of $71.5 \%$. While the sensitivity for the distress score when setting 46 or higher as the cutoff point was $90.3 \%$, the corresponding specificity reached $81.2 \%$. 
Table 2

Accuracy of PQ-B total score and distress score in screening CHR

\begin{tabular}{|c|c|c|c|c|c|c|c|}
\hline PQ-B cutoff & $\begin{array}{l}\text { Sensitivity } \\
\text { (\%) }\end{array}$ & $\begin{array}{l}\text { Specificity } \\
(\%)\end{array}$ & $\mathrm{Yl}^{\mathrm{a}}$ & $\underset{a}{\mathrm{LR}+}$ & AUC $^{a}$ & $95 \% \mathrm{Cl}$ & $p$ \\
\hline \multicolumn{8}{|l|}{ Total score } \\
\hline$\geq 10$ & 94.4 & 61.9 & 0.56 & 2.48 & 0.842 & $\begin{array}{l}0.806- \\
0.877\end{array}$ & $<0.001$ \\
\hline$\geq 11$ & 91.7 & 65.6 & 0.57 & 2.67 & 0.842 & $\begin{array}{l}0.806- \\
0.877\end{array}$ & $<0.001$ \\
\hline$\geq 12$ & 91.7 & 68.9 & 0.61 & 2.95 & 0.842 & $\begin{array}{l}0.806- \\
0.877\end{array}$ & $<0.001$ \\
\hline$\geq 13^{b}$ & 91.7 & 71.5 & 0.63 & 3.22 & 0.842 & $\begin{array}{l}0.806- \\
0.877\end{array}$ & $<0.001$ \\
\hline$\geq 14$ & 87.5 & 74.1 & 0.62 & 3.38 & 0.842 & $\begin{array}{l}0.806- \\
0.877\end{array}$ & $<0.001$ \\
\hline$\geq 15$ & 86.1 & 76.3 & 0.62 & 3.63 & 0.842 & $\begin{array}{l}0.806- \\
0.877\end{array}$ & $<0.001$ \\
\hline \multicolumn{8}{|l|}{$\begin{array}{l}\text { Distress } \\
\text { score }\end{array}$} \\
\hline$\geq 43$ & 90.3 & 78.2 & 0.69 & 4.14 & 0.900 & $\begin{array}{l}0.863- \\
0.937\end{array}$ & $<0.001$ \\
\hline$\geq 44$ & 90.3 & 79.2 & 0.70 & 4.34 & 0.900 & $\begin{array}{l}0.863- \\
0.937\end{array}$ & $<0.001$ \\
\hline$\geq 45$ & 90.3 & 80.3 & 0.71 & 4.58 & 0.900 & $\begin{array}{l}0.863- \\
0.937\end{array}$ & $<0.001$ \\
\hline$\geq 46^{\mathrm{b}}$ & 90.3 & 81.2 & 0.72 & 4.80 & 0.900 & $\begin{array}{l}0.863- \\
0.937\end{array}$ & $<0.001$ \\
\hline$\geq 47$ & 83.3 & 82.2 & 0.66 & 4.68 & 0.900 & $\begin{array}{l}0.863- \\
0.937\end{array}$ & $<0.001$ \\
\hline$\geq 48$ & 79.2 & 83.0 & 0.62 & 4.66 & 0.900 & $\begin{array}{l}0.863- \\
0.937\end{array}$ & $<0.001$ \\
\hline \multicolumn{8}{|c|}{ a YI: Youden's index; LR+: positive likelihood ratio; AUC: area under the curve } \\
\hline
\end{tabular}

\subsubsection{The significant items of PQ-B in detecting CHR}

To investigate which items were significant in detecting CHR in Chinese college students, independentsamples t-tests was initially performed to compare the differences in the item scores of $\mathrm{PQ}-\mathrm{B}$ between $\mathrm{CHR}$ and non-CHR individuals. The results demonstrated that the score for each individual item in PQ-B 
was significantly higher in the CHR group (all $p<0.001$ ), which was presented in a bar chart (see Fig. 3). Furthermore, a Spearman correlation analysis showed that higher item scores of PQ-B were significantly associated with the diagnosis of CHR (all $p<0.001)$. Therefore, we did a conditional backward logistic regression analysis to explore the key items involved in the detection of CHR subjects. All PQ-B item scores were set as the independent variables and the diagnosis (CHR or non-CHR) was set as the dependent variable. As presented in Table 3, 8 items (PQ1, PQ3, PQ4, PQ12, PQ13, PQ15, PQ17 and PQ18) were entered the regression model.

Table 3

Logistic regression of the $\mathrm{CHR} /$ non-CHR classification by $\mathrm{PQ}-\mathrm{B}$ items

\begin{tabular}{|lllllll|}
\hline Variables & B & S.E. & Wald & Df & $\mathbf{p}$ & Exp(B) \\
\hline PQ1 & 0.223 & 0.086 & 6.764 & 1 & 0.009 & 1.249 \\
\hline PQ4 & 0.225 & 0.077 & 8.489 & 1 & 0.004 & 1.252 \\
\hline PQ12 & 0.240 & 0.094 & 6.504 & 1 & 0.011 & 1.271 \\
\hline PQ13 & 0.204 & 0.082 & 6.138 & 1 & 0.013 & 1.226 \\
\hline PQ15 & 0.282 & 0.103 & 7.527 & 1 & 0.006 & 1.326 \\
\hline PQ17 & 0.182 & 0.088 & 4.317 & 1 & 0.038 & 1.200 \\
\hline PQ18 & 0.199 & 0.064 & 9.666 & 1 & 0.002 & 1.220 \\
\hline PQ21 & 0.188 & 0.094 & 3.995 & 1 & 0.046 & 1.206 \\
\hline Constant & -9.813 & 0.468 & 439.389 & 1 & $<0.001$ & 0.000 \\
\hline Overall model fit test & $\chi^{2}=252.494, p<0.001 ;$ Hosmer-Lemeshow test value $=8.309, p=0.306$. \\
\hline Associated strength & Cox-Snell $\mathrm{R}^{2}=0.011 ;$ Nagelkerke $\mathrm{R}^{2}=0.263$ & & \\
\hline
\end{tabular}

\section{Discussion}

Detection of CHR samples in both the clinical [10,33-36] and non-clinical settings[31, 37, 38] had caught great attention across the world, while the detection rate of CHR subjects in those studies varied greatly. Previous studies reported that the prevalence of CHR in the clinician-referred samples was 32-42\%[3335], which were roughly consistent with each other. While the detection rate in samples of help-seeking individuals ranged from $4.2-80 \%$, which differed from site to site[10,39-41]. As for the general population (primarily focused on young adults and adolescences), the annual incidence of new cases of CHR was estimated to be $1 / 10000$ [33], and the prevalence was reported to range from $0.3-2.4 \%[31,37$, 38]. The diagnostic instruments were considered to be a great factor contributing to the inconsistency of the detection rate. Schultze et al.[31] screened CHR in a young adult community with SIPS and SPI-A, and reported that $2.4 \%$ of the participants meet at least one criterion of $\mathrm{CHR}$, while only $0.6 \%$ met the criteria of APS identified by SIPS. Salazar et al.[42] reviewed 56 studies and suggested that the $0.3 \%$ of the 
general non-help-seeking young population met the DSM-5-APS criteria, while $1.3 \%$ of the general population met the SIPS-APSS criteria. In this study, the detection rate of CHR subjects identified with the SIPS criteria in Chinese college students was $0.3 \%$. To our knowledge, this is the first multicenter epidemiological study on the detection of CHR subjects in Chinese college students. The participants were recruited from three different cities, including highly developed cities like Shanghai and Nanjing, and also a second-tier city like Nanchang, through a random sampling way. Also, the colleges involved included both general higher educational institutions and vocational colleges. Therefore, the sample in the present study was representative for Chinese college students. Although the detection rate reported in our study was lower than $1.1 \%$ reported in a single-centered study conducted in Chinese college students[38], but was generally in line with other studies of this kind mentioned above.

Since first-episode psychosis usually occur in late adolescence and early adulthood, CHR or prodromal psychosis were believed to have an earlier onset $[43,44]$. Our research demonstrated that the peak age range of the CHR identification by SIPS was between 17 to 20 years in the sample of Chinese college students, which was largely in line with previous studies but a little bit narrower (e.g. SHARP reported 16 to 21 years in a Chinese sample) $[10,27]$. The mean age of CHR was $18.3(S D=1.5)$ years in this sample, which was similar to the sample of NAPLS[33], but younger than that of SHARP[10], and older than that of RAP[27]. The demographic features of recruited participants in these studies may account for the age discrepancy. As the participants recruited in this study were college students with an age range of 14 to 31 years, while NAPLS recruited clinician-referred subjects aged 12 to 35 years, SHARP included helpseeking subjects aged 15 to 45 years, and RAP included help-seeking subjects aged 12 to 22 years.

Similar to previous studies using PQ-B to screen CHR in Chinese population[16, 22, 45-47], our research proved that $P Q-B$ could be a feasible first-step instrument for screening suspected $C H R$ subjects in college students. Both the sensitivity and specificity of PQ-B were excellent in this study. Also, the results showed that 8 items of PQ-B including the PQ1 (asking "Do familiar surroundings sometimes seem strange, confusing, threatening or unreal to you?"), the PQ3 (asking "Do things that you see appear different from the way they usually do (brighter or duller, larger or smaller, or changed in some other way)?"), the PQ4 (asking "Have you had experiences with telepathy, psychic forces, or fortune telling?"), the PQ12 (asking "Do you worry at times that something may be wrong with your mind?"), the PQ13 (asking "Have you ever felt that you don't exist, the world does not exist, or that you are dead?"), the PQ15 (asking "Do you hold beliefs that other people would find unusual or bizarre?"), the PQ17 (asking "Are your thoughts sometimes so strong that you can almost hear them?") and the PQ18 (asking "Do you find yourself feeling mistrustful or suspicious of other people?") had significant predictive value of SIPS diagnosis, which indicated that delusional ideas, perceptual abnormalities and suspiciousness were key symptoms in identifying CHR subjects. Similarly, Xu et al.[22] and Schultze et al.[31] also reported that perceptual abnormalities, delusional ideas and suspiciousness were the most prevalent psychosis-risk symptoms.

Nevertheless, our results recommended higher cut-off points for the total score and distress score of PQ-B (13 and 46) than those of SHARP did in the Chinese population (7 and 24)[22], which were also much higher than the cut-off points reported in the initial validation study of PQ-B (3 and 6)[16]. Despite the 
cultural background and language may lead to the differences of PQ-B cut-off points[48], there must still be other reason underlying the differences between the present study and SHARP. First, the participants in this study were college students who were tested with PQ-B in non-clinical settings (online or in the colleges), while SHARP recruited help-seeking out-patients from a psychological counselling center[22]. As prodromal symptoms mentioned in PQ-B were often mixed with psychotic-like experiences (PLEs) that commonly existed in the general population[49-52], subjects who only had PLEs may also been tested positive with $\mathrm{PQ}-\mathrm{B}$, causing false positives. Also, being rated in a clinical setting, rather than a non-clinical setting, may help the participants better understand the prodromal symptoms mentioned in the scale, and thus reducing false positive errors. Indeed, if we used the same cut-off points as SHARP did in their screening procedure, $72.0 \%(16595 / 23063)$ of the participants would be screened positive with PQ-B in this study, while the positive rate of PQ-B in SHARP was 62.1\% (1681/2705)[10], which may indicate that our false positive rate in PQ-B screening was higher. Second, the screening procedure differed. SHARP employed a 2-stage method where the subjects tested positive with PQ-B would all be invited to the SIPS interview, and $18.3 \%$ of the subjects tested negative with PQ-B were also interviewed[10]. However, we used a 3-stage method that applied a telephone interview to subjects screened as positive with PQ-B and only subjects screened as positive in the telephone interview were invited to the SIPS interview. Moreover, further investigations were not provided to the negative subjects in the present study. These variance in the screening procedure may have contributed to the underestimation of CHR subjects, which would interfere the examination of the psychometric properties of PQ-B.

As discussed in the last paragraph, the telephone assessment may cause the missed diagnosis of CHR, which was a limitation of this study, whereas, it saved a lot of human resources in the process of CHR screening, indeed, particularly in a large general population. It is worth mentioning that the telephone assessment employed in this study was not a semi-structured or structured interview that can be seen in other studies, although we also use SIPS as the assessing instrument[31, 53, 54]. In the present study, it only required the researchers to ask questions following the subscale for positive symptoms, and rate the severity for each domain according to the subjective reports from the participants. Therefore, it only took 5 minutes in average, which was much time-saving compared to a structured/semi-structured interview. In fact, it also enhanced the screening efficiency by having precluded almost $90 \%$ of the subjects tested positive with PQ-B from receiving a face-to-face SIPS interview, despite which was, of course, based on the sacrifice of the detection rate.

Another limitation is that, due to resource limitations, the information obtained from the participants was incomplete. On one hand, we missed some important clinical characteristics of the subjects, including family history and psychiatric comorbidity. On the other hand, the CHR subjects were not followed after being diagnosed, and thus, the transition to psychosis or other mental disorders was not investigated in this study.

\section{Conclusions}


In conclusion, the 3-stage method is a useful strategy in screening CHR individuals in general population, especially when the population is large. Among college students, those with experiences of delusional ideas, perceptual abnormalities or suspiciousness were more likely to be screened as CHR subjects. For future researches, a 1- to 2-year follow-up of the CHR subjects is essential to investigate the conversion of CHR subjects, and to explore the related risk factors.

\section{Abbreviations}

CHR

Clinical high risk

UHR

Ultra-high risk

$P Q-B$

The Prodromal Questionnaire-brief version

SOPS

The Scale of Prodromal Symptoms

SIPS

The Structured Interview for Prodromal Syndromes

CAARMS

The Comprehensive Assessment of At-Risk Mental States

SPI-A

The Schizophrenia Proneness Instrument, Adult

SPI-CY

The Schizophrenia Proneness Instrument, Child \& Youth version

PQ-16

The Prodromal Questionnaire-16 items (PQ-16)

EPS

The Early Psychosis Screener

PLEs

Psychotic-like experiences

SMHC

Shanghai Mental Health Centre

$\mathrm{TJH}$

Tongji Hospital of Tongji University

$\mathrm{NBH}$

Nanjing Brain Hospital

FAHNU

The First Affiliated Hospital of Nanchang University

APSS

Attenuated Positive Symptom Syndrome 
BIPS

Brief Intermittent Psychotic Syndrome

GRDS

Genetic Risk and Deterioration Syndrome

ROC

Receiver operating characteristic

AUCs

Areas under the curve

SHARP

The Shanghai-At-Risk-for-Psychosis Study

NAPLS

पThe North American Prodrome Longitudinal Study

RAP

The Recognition and Prevention Program

\section{Declarations}

\section{Ethics approval and consent to participate}

This study was approved by the Research Ethics Committee at the Shanghai Mental Health Centre (SMHC), and carried out in accordance with the Declaration of Helsinki. Written informed consent was received from all applicable participants prior to inclusion.

\section{Consent to publish}

Not applicable.

\section{Availability of data and materials}

The dataset(s) generated during the current study are not publically available due to ethical restrictions but are available from the corresponding author on reasonable request.

\section{Competing interests}

The authors declare no competing interest.

\section{Funding}

This study was supported by grants from Ministry of Science and Technology of China (2016YFC1306805), Priority of Shanghai Key Discipline of Medicine (2017ZZ02020), National Natural Science Foundation of China (81974214), Clinical Research Plan of Shanghai Shen Kang Hospital 
Development Center (16CR1032B). The funder had no role in the design, collection, management, analysis, and approval of manuscript or decision to submit the manuscript for publication.

\section{Authors' contributions}

Z.L. conceived of the study. XY.L., F.L., AS.Q., SP.X., MR.H., TH.Z., JJ.W. and Z.L. contributed to the design of the study. JX.W., XY.L., F.L., AS.Q., Q.C., XF.G., Q.Z., YH.Y., JY.S., W.Y., X.Y., J.T. and SL.W. contributed to the patient recruitment and data acquisition. JX.W. contributed to the data interpretation and statistical analysis, as well as the drafting of the paper. XY.L. and Z.L. revised the manuscript critically. All the authors read and approved the final manuscript.

\section{Acknowledgements}

We gratefully acknowledged the participation of all individuals involved in the study.

\section{References}

1. Sullivan HS. The onset of schizophrenia. 1927. Am J Psychiatry. 1994;151(6 Suppl):134-9.

2. McGlashan TH, Johannessen JO. Early detection and intervention with schizophrenia: rationale. Schizophr Bull. 1996;22(2):201-22.

3. Seidman LJ, Nordentoft M. New Targets for Prevention of Schizophrenia: Is It Time for Interventions in the Premorbid Phase? Schizophr Bull. 2015;41(4):795-800.

4. Lieberman JA, Small SA, Girgis RR. Early Detection and Preventive Intervention in Schizophrenia: From Fantasy to Reality. Am J Psychiatry. 2019;176(10):794-810.

5. Perkins DO, Gu H, Boteva K, Lieberman JA. Relationship between duration of untreated psychosis and outcome in first-episode schizophrenia: a critical review and meta-analysis. Am J Psychiatry. 2005;162(10):1785-804.

6. McGorry PD, Yung AR, Phillips LJ. The "close-in" or ultra high-risk model: a safe and effective strategy for research and clinical intervention in prepsychotic mental disorder. Schizophr Bull. 2003;29(4):771-90.

7. Cornblatt B, Lencz T, Obuchowski M. The schizophrenia prodrome: treatment and high-risk perspectives. Schizophr Res. 2002;54(1-2):177-86.

8. Fusar-Poli P. The Clinical High-Risk State for Psychosis (CHR-P), Version II. Schizophr Bull. 2017;43(1):44-7.

9. Fusar-Poli P, Bonoldi I, Yung AR, Borgwardt S, Kempton MJ, Valmaggia L, Barale F, Caverzasi E, McGuire P. Predicting psychosis: meta-analysis of transition outcomes in individuals at high clinical risk. Arch Gen Psychiatry. 2012;69(3):220-9. 
10. Zhang T, Li H, Woodberry KA, Seidman LJ, Zheng L, Li H, Zhao S, Tang Y, Guo Q, Lu X, et al. Prodromal psychosis detection in a counseling center population in China: an epidemiological and clinical study. Schizophr Res. 2014;152(2-3):391-9.

11. Schultze-Lutter F, Michel C, Schmidt SJ, Schimmelmann BG, Maric NP, Salokangas RK, RiecherRossler A, van der Gaag M, Nordentoft M, Raballo A, et al. EPA guidance on the early detection of clinical high risk states of psychoses. Eur Psychiatry. 2015;30(3):405-16.

12. Miller TJ, McGlashan TH, Rosen JL, Cadenhead K, Cannon T, Ventura J, McFarlane W, Perkins DO, Pearlson GD, Woods SW. Prodromal assessment with the structured interview for prodromal syndromes and the scale of prodromal symptoms: predictive validity, interrater reliability, and training to reliability. Schizophr Bull. 2003;29(4):703-15.

13. Yung AR, Yuen HP, McGorry PD, Phillips LJ, Kelly D, Dell'Olio M, Francey SM, Cosgrave EM, Killackey E, Stanford C, et al. Mapping the onset of psychosis: the Comprehensive Assessment of At-Risk Mental States. Aust N Z J Psychiatry. 2005;39(11-12):964-71.

14. Schultze-Lutter F, Ruhrmann S. tter: Development and evaluation of the schizophrenia proneness instrument, adult version (SPI-A). Schizophr Res. 2006;86(06):4-5.

15. Fux L, Walger P, Schimmelmann BG, Schultze-Lutter F. The Schizophrenia Proneness Instrument, Child and Youth version (SPI-CY): Practicability and discriminative validity. Schizophr Res. 2013;146(1-3):69-78.

16. Loewy RL, Pearson R, Vinogradov S, Bearden CE, Cannon TD. Psychosis risk screening with the Prodromal Questionnaire-brief version (PQ-B). Schizophr Res. 2011;129(1):42-6.

17. Ising HK, Veling W, Loewy RL, Rietveld MW, Rietdijk J, Dragt S, Klaassen RM, Nieman DH, Wunderink $\mathrm{L}$, Linszen $\mathrm{DH}$, et al. The validity of the 16-item version of the Prodromal Questionnaire (PQ-16) to screen for ultra high risk of developing psychosis in the general help-seeking population. Schizophr Bull. 2012;38(6):1288-96.

18. Miller T, Cicchetti D, Mcglashan T, Woods S. Brief self-report screen to detect the schizophrenia prodrome. In: 12th Biennial Winter Workshop on Schizophrenia: 2004; 2004: 35-35.

19. Brodey BB, Addington J, First MB, Perkins DO, Woods SW, Walker EF, Walsh B, Nieri JM, Nunn MB, Putz J, et al. The Early Psychosis Screener (EPS): Item development and qualitative validation. Schizophr Res. 2018;197:504-8.

20. Brodey BB, Girgis RR, Favorov OV, Addington J, Perkins DO, Bearden CE, Woods SW, Walker EF, Cornblatt BA, Brucato G, et al. The Early Psychosis Screener (EPS): Quantitative validation against the SIPS using machine learning. Schizophr Res. 2018;197:516-21.

21. Li-Na Z, Ji-Jun W, Tian-Hong Z, Hui L, Chun-Bo L. JIANG K-D: Reliability and validity of the Chinese version of Scale of Psychosis-risk Symptoms. Chinese Mental Health Journal. 2012;26(8):571-6.

22. Xu L, Zhang T, Zheng L, Li H, Tang Y, Luo X, Sheng J, Wang J. Psychometric Properties of Prodromal Questionnaire-Brief Version among Chinese Help-Seeking Individuals. PLoS One. 2016;11(2):e0148935. 
23. Chen F, Wang L, Heeramun-Aubeeluck A, Wang J, Shi J, Yuan J, Zhao X. Identification and characterization of college students with attenuated psychosis syndrome in China. Psychiatry Res. 2014;216(3):346-50.

24. Guo Y, Qu S, Qin H. Study of the relationship between self-stigma and subjective quality of life for individuals with chronic schizophrenia in the community. Gen Psychiatr. 2018;31(3):e100037.

25. Zhang T, Xu L, Tang Y, Li H, Tang X, Cui H, Wei Y, Wang Y, Hu Q, Liu X, et al. Prediction of psychosis in prodrome: development and validation of a simple, personalized risk calculator. Psychol Med. 2019;49(12):1990-8.

26. Zhang T, Li H, Tang Y, Li H, Zheng L, Guo Q, Zhao S, Zhuo K, Qian Z, Wang L, et al. Screening schizotypal personality disorder for detection of clinical high risk of psychosis in Chinese mental health services. Psychiatry Res. 2015;228(3):664-70.

27. Cornblatt BA, Carrion RE, Auther A, McLaughlin D, Olsen RH, John M, Correll CU. Psychosis Prevention: A Modified Clinical High Risk Perspective From the Recognition and Prevention (RAP) Program. Am J Psychiatry. 2015;172(10):986-94.

28. Sun M, Hu X, Zhang W, Guo R, Hu A, Mwansisya TE, Zhou L, Liu C, Chen X, Huang X, et al. Psychoticlike experiences and associated socio-demographic factors among adolescents in China. Schizophr Res. 2015;166(1-3):49-54.

29. Wang C, Wang Q, Li X, Zhang Y, Wei W, Deng W, Guo W, He L, Tang W, Chen T, et al. Rural birth/upbringing and childhood adversities are associated with psychotic experiences in university students in China. Schizophr Res. 2019;209:105-12.

30. Loewy RL, Bearden CE, Johnson JK, Raine A, Cannon TD. The prodromal questionnaire (PQ): preliminary validation of a self-report screening measure for prodromal and psychotic syndromes. Schizophr Res. 2005;79(1):117-25.

31. Schultze-Lutter F, Michel C, Ruhrmann S, Schimmelmann BG. Prevalence and clinical relevance of interview-assessed psychosis-risk symptoms in the young adult community. Psychol Med. 2018;48(7):1167-78.

32. Huang Y, Wang Y, Wang H, Liu Z, Yu X, Yan J, Yu Y, Kou C, Xu X, Lu J, et al. Prevalence of mental disorders in China: a cross-sectional epidemiological study. Lancet Psychiatry. 2019;6(3):211-24.

33. Addington J, Cadenhead KS, Cannon TD, Cornblatt B, McGlashan TH, Perkins DO, Seidman LJ, Tsuang M, Walker EF, Woods SW, et al. North American Prodrome Longitudinal Study: a collaborative multisite approach to prodromal schizophrenia research. Schizophr Bull. 2007;33(3):665-72.

34. Fusar-Poli P, Byrne M, Badger S, Valmaggia LR, McGuire PK. Outreach and support in south London (OASIS), 2001-2011: ten years of early diagnosis and treatment for young individuals at high clinical risk for psychosis. Eur Psychiatry. 2013;28(5):315-26.

35. Broome MR, Woolley JB, Johns LC, Valmaggia LR, Tabraham P, Gafoor R, Bramon E, McGuire PK. Outreach and support in south London (OASIS): implementation of a clinical service for prodromal psychosis and the at risk mental state. Eur Psychiatry. 2005;20(5-6):372-8. 
36. Yung AR, Nelson B, Stanford C, Simmons MB, Cosgrave EM, Killackey E, Phillips LJ, Bechdolf A, Buckby J, McGorry PD. Validation of "prodromal" criteria to detect individuals at ultra high risk of psychosis: 2 year follow-up. Schizophr Res. 2008;105(1-3):10-7.

37. Schultze-Lutter F, Michel C, Ruhrmann S, Schimmelmann BG. Prevalence and clinical significance of DSM-5-attenuated psychosis syndrome in adolescents and young adults in the general population: the Bern Epidemiological At-Risk (BEAR) study. Schizophr Bull. 2014;40(6):1499-508.

38. Wang L, Shi J, Chen F, Yao Y, Zhan C, Yin X, Fang X, Wang H, Yuan J, Zhao X. Family Perception and 6-Month Symptomatic and Functioning Outcomes in Young Adolescents at Clinical High Risk for Psychosis in a General Population in China. PLoS One. 2015;10(9):e0138361.

39. Gerstenberg M, Theodoridou A, Traber-Walker N, Franscini M, Wotruba D, Metzler S, Muller M, Dvorsky D, Correll CU, Walitza S, et al. Adolescents and adults at clinical high-risk for psychosis: age-related differences in attenuated positive symptoms syndrome prevalence and entanglement with basic symptoms. Psychol Med. 2016;46(5):1069-78.

40. Fusar-Poli P, De Micheli A, Cappucciati M, Rutigliano G, Davies C, Ramella-Cravaro V, Oliver D, Bonoldi I, Rocchetti M, Gavaghan L, et al. Diagnostic and Prognostic Significance of DSM-5 Attenuated Psychosis Syndrome in Services for Individuals at Ultra High Risk for Psychosis. Schizophr Bull. 2018;44(2):264-75.

41. Spada G, Molteni S, Pistone C, Chiappedi M, McGuire P, Fusar-Poli P, Balottin U. Identifying children and adolescents at ultra high risk of psychosis in Italian neuropsychiatry services: a feasibility study. Eur Child Adolesc Psychiatry. 2016;25(1):91-106.

42. Salazar de Pablo G, Catalan A, Fusar-Poli P. Clinical Validity of DSM-5 Attenuated Psychosis Syndrome: Advances in Diagnosis, Prognosis, and Treatment. JAMA Psychiatry 2019.

43. Welham JL, Thomis RJ, McGrath JJ. Age-at-first-registration for affective psychosis and schizophrenia. Schizophr Bull. 2004;30(4):849-53.

44. Chan V. Schizophrenia and Psychosis: Diagnosis, Current Research Trends, and Model Treatment Approaches with Implications for Transitional Age Youth. Child Adolesc Psychiatr Clin N Am. 2017;26(2):341-66.

45. Fonseca-Pedrero E, Inchausti F, Perez-Albeniz A, Ortuno-Sierra J. Validation of the Prodromal Questionnaire-Brief in a representative sample of adolescents: Internal structure, norms, reliability, and links with psychopathology. Int J Methods Psychiatr Res. 2018;27(4):e1740.

46. Kline E, Thompson E, Demro C, Bussell K, Reeves G, Schiffman J. Longitudinal validation of psychosis risk screening tools. Schizophr Res. 2015;165(2-3):116-22.

47. Jang YE, Lee TY, Hur JW, Kwon JS. Validation of the Korean Version of the Prodromal QuestionnaireBrief Version in Non-Help-Seeking Individuals. Psychiatry Investig. 2019;16(2):109-14.

48. Cicero DC, Krieg A, Martin EA. Measurement Invariance of the Prodromal Questionnaire-Brief Among White, Asian, Hispanic, and Multiracial Populations. Assessment. 2019;26(2):294-304.

49. Fonseca-Pedrero E, Gooding DC, Ortuno-Sierra J, Paino M. Assessing self-reported clinical high risk symptoms in community-derived adolescents: A psychometric evaluation of the Prodromal 
Questionnaire-Brief. Compr Psychiatry. 2016;66:201-8.

50. Shevlin M, Murphy J, Dorahy MJ, Adamson G. The distribution of positive psychosis-like symptoms in the population: a latent class analysis of the National Comorbidity Survey. Schizophr Res. 2007;89(1-3):101-9.

51. Rossler W, Riecher-Rossler A, Angst J, Murray R, Gamma A, Eich D, van Os J, Gross VA. Psychotic experiences in the general population: a twenty-year prospective community study. Schizophr Res. 2007;92(1-3):1-14.

52. Sullivan SA, Kounali D, Cannon M, David AS, Fletcher PC, Holmans P, Jones H, Jones PB, Linden DEJ, Lewis G, et al. A Population-Based Cohort Study Examining the Incidence and Impact of Psychotic Experiences From Childhood to Adulthood, and Prediction of Psychotic Disorder. Am J Psychiatry. 2020;177(4):308-17.

53. Schultze-Lutter F, Schimmelmann BG, Fluckiger R, Michel C. Effects of age and sex on clinical highrisk for psychosis in the community. World J Psychiatry. 2020;10(5):101-24.

54. Michel C, Schmidt SJ, Schnyder N, Fluckiger R, Kaufeler I, Schimmelmann BG, Schultze-Lutter F. Associations of psychosis-risk symptoms with quality of life and self-rated health in the Community. Eur Psychiatry. 2019;62:116-23.

\section{Figures}

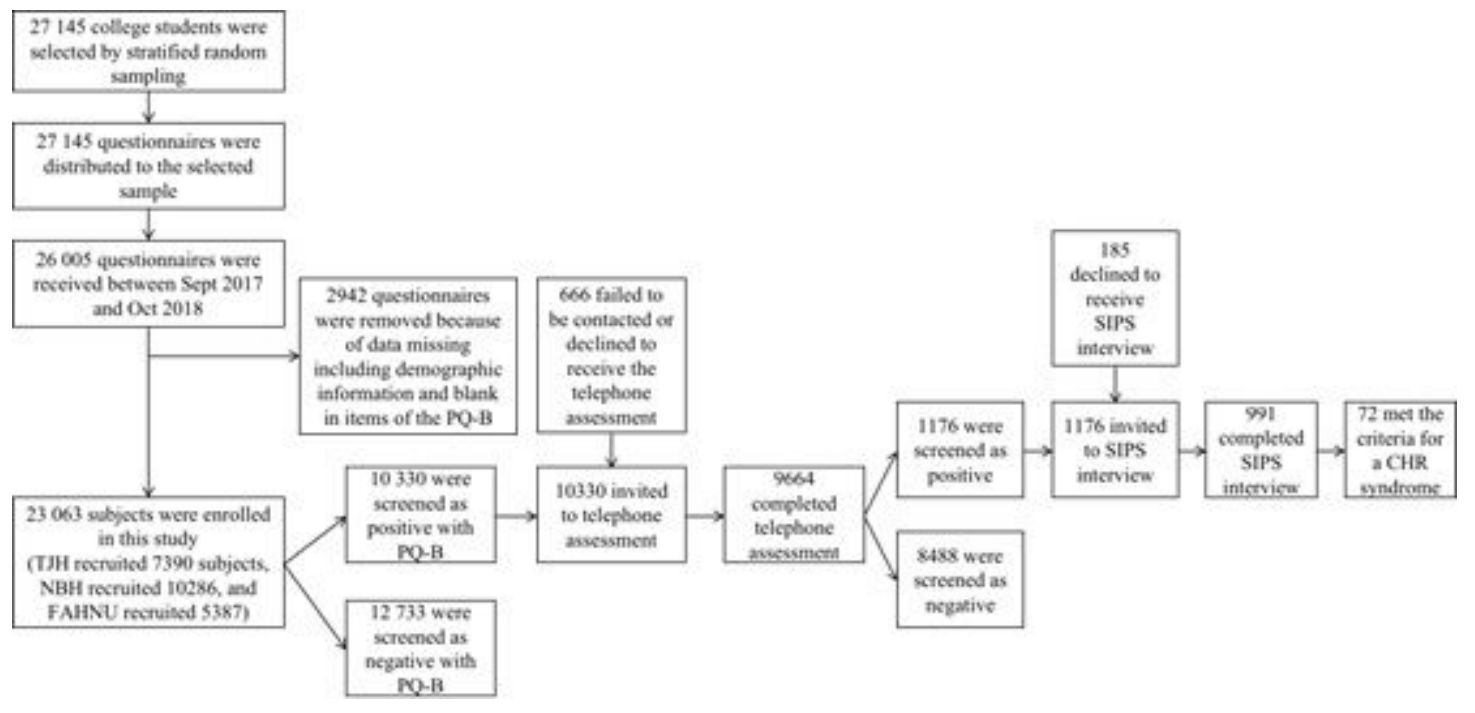

\section{Figure 1}

Sample flowchart (TJH: Tongji Hospital of Tongji University; NBH: Nanjing Brain Hospital; FAHNU: The First Affiliated Hospital of Nanchang University) 

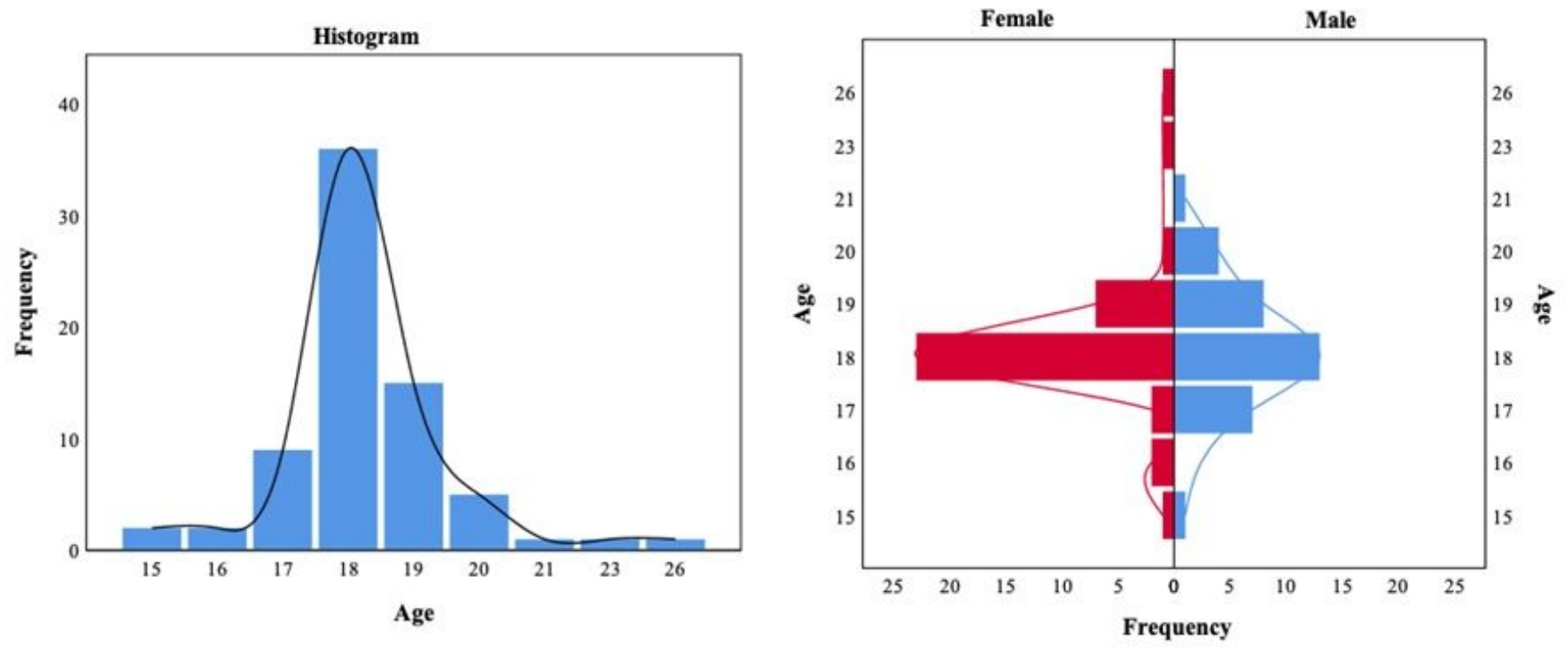

Figure 2

Age distribution for the total sample, females and males meeting CHR criteria

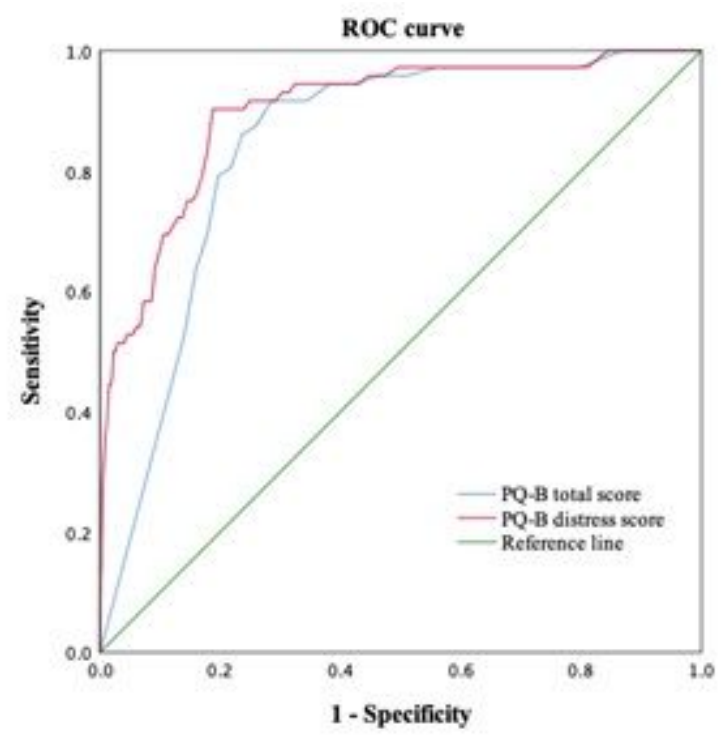

Figure 3

ROC curves for the total score and distress score of PQ-B as indicator of CHR diagnosis 


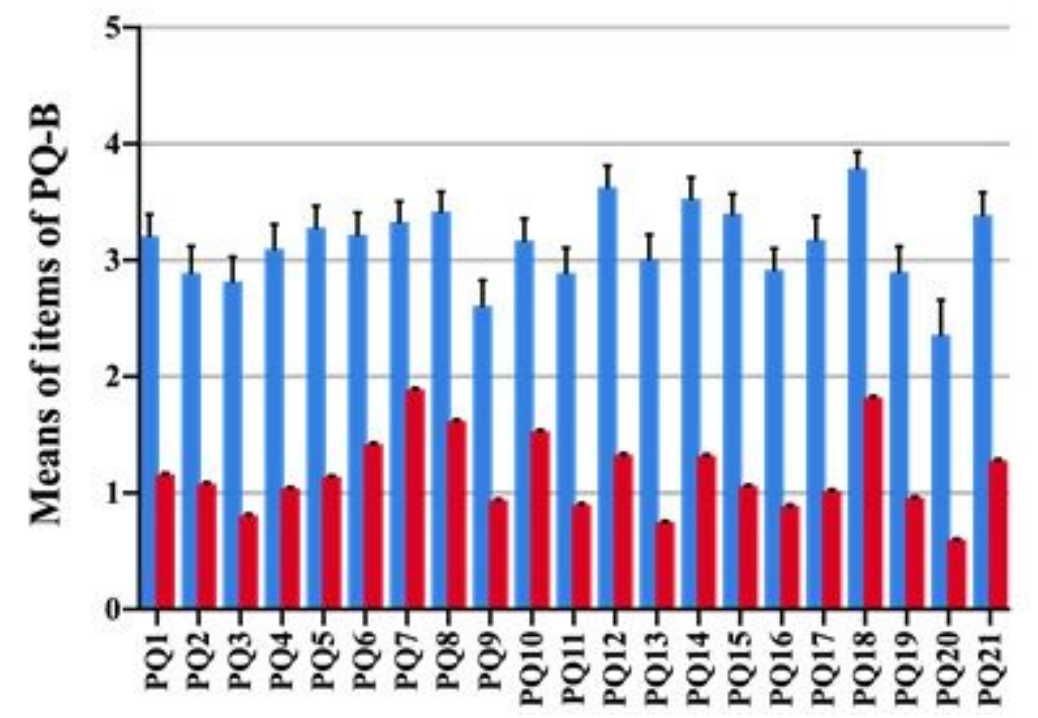

\section{CHR subjects}

non-CHR subjects

\section{Figure 4}

Comparison of $\mathrm{PQ}-\mathrm{B}$ item scores in $\mathrm{CHR}$ and non-CHR individuals: the means of $\mathrm{PQ}-\mathrm{B}$ items are significantly higher in CHR individuals $(p<0.001)$

\section{Supplementary Files}

This is a list of supplementary files associated with this preprint. Click to download.

- supplements.docx 